\title{
Effect of Telmisartan Versus Captopril on Insulin Resistance in Hypertensive Type 2 Diabetic Patients
}

\author{
Adnan Ali Zainal *
}

Fadhel Abbas Al-Hamamy **

\begin{abstract}
Background and objectives: Hypertension is an extremely common co-morbid condition in diabetes. Peripheral insulin resistance (IR) plus compensatory hyperinsulinaemia is a common mechanism underlying both hypertension and diabetes. The aim of this study was to compare the effects of telmisartan and captopril on IR in type 2 diabetes mellitus with hypertension.

Materials and Methods: This study was conducted in the Department of Pharmacology, Mosul College of Medicine, University of Mosul and Al-Wafaa center for diabetes management, Mosul, Iraq, from 1 March, 2012 to 30 March, 2013. Sixty-eight patients on oral hypoglycemic agents were assigned to receive two month treatment of either telmisartan $(n=34)$ or captopril $(n=34)$. Forty diabetic normotensive patients, with age and BMI, matched to the diabetic hypertensive patients, served as a control group. The oral hypoglycemic agents remained unchanged during the two- months study period. Insulin resistance was estimated by homeostasis model assessment of insulin resistance (HOMA-IR) and quantitative insulin sensitivity check index (QUICKI).
\end{abstract}

Results: This study showed that the two groups of diabetic hypertensive patients who were assigned to receive captopril or telmisartan have a non-significant difference in fasting serum glucose (FSG), fasting serum insulin (FSI), QUICKI and HOMA- IR before starting therapy. Telmisartan and captopril groups showed insignificant change in body mass index (BMI). HOMA-IR, QUICKI, FSI and FSG were changed non-significantly in telmisartan group; while FSI, FSG and HOMA-IR decreased significantly (from $18.1 \pm 9.69$ to $15.14 \pm 7.49, \mathrm{p}=0.022$; and from $187.32 \pm 65.34$ to $162.95 \pm 56.87, \mathrm{p}=0.048$; and from $8.27 \pm 6.28$ to $6.02 \pm 3.73$, p-value $=0.027$, respectively) and QUICKI increased significantly (from 0.293 \pm 0.024 to $0.306 \pm 0.031, \mathrm{p}$-value $=0.022$ ) in the captopril group versus baseline.

Conclusion: Captopril, but not telmisartan, significantly improves insulin sensitivity in diabetic hypertensive patients.

Keywords: Captopril, HOMA-IR, telmisartan, type 2 diabetes.

\section{INTRODUCTION}

The World Health Organization (WHO) estimated that there were 30 million people who had diabetes worldwide in 1985. This number increased to 135 million by 1995 and reached 217 million in 2005. By the year 2030 WHO predicts this number will increase to at least 366 million (Matheus et al., 2013). In a recent study in Iraq, hypertension (HT) was seen in $\sim 90 \%$ of diabetic patients (in Basrah), and approximately half (48\%) of them were achieving a target BP $<130 / 80 \mathrm{mmHg}$. (Mansour, 2012)

Hypertension is an extremely common co-morbid condition in diabetes, in most diabetic populations, about $80 \%$ have coexisting HT and, vice versa, about $70 \%$ of patients with HT do have or will develop type 2 diabetes mellitus
(T2DM) (Standl et al.,2012), depending on obesity, ethnicity and age. In T2DM, HT is often present as a part of the metabolic syndrome of Insulin resistance (IR), including central obesity and dyslipidaemia. (Patel and Mehta, 2013, Schutta, 2007).

Insulin resistance describes an impaired biological response to insulin. (Mcfarlane et al., 2001), it is a state in which insulin-sensitive tissues (liver, skeletal muscle and adipose tissue) exhibit an inadequate response to normal circulating levels of insulin, (Savage et al., 2005; Savage et al., 2007), or fail to respond to normal levels of circulating insulin (Olivares-Reyes et al., 2009). Clinically, the term "insulin resistance" implies that higher-than-normal concentrations of insulin are required to maintain normoglycemia (Saini, 2010).

\footnotetext{
* Lecturer / College of Pharmacy/University of Mosul/E-mail: sinaiexplorer2000@yahoo.com.

** Assistant Professor / Mosul College of Medicine/ University of Mosul.
} 
Peripheral IR plus compensatory hyperinsulinaemia is a common mechanism underlying both HT and T2DM (Östergren, 2007).

Captopril is a selective inhibitor of angiotensin converting enzyme (ACEI). (Ibrahim, et al., 2010). ACE inhibitors prevent the conversion of angiotensin I (Ang I) to Ang II by inhibiting ACEI-enzyme complex (Cohen and Townsend, 2008), thus reduce the formation of Ang II. By blocking the degradation of the vasoactive peptide bradykinin, thus promoting the release of nitric oxide, vasodilation, and natriuresis. (Berra and Miller 2009).

There are some Angiotensin receptor blockers (ARBs) that can function as a partial agonist of peroxisome proliferator-activated receptor gamma (PPAR- $\gamma$ ) and improve carbohydrate and lipid metabolism, even in the absence of a functional Ang II receptor. Telmisartan has PPAR- $\gamma$ activity at therapeutically achievable doses that may result in a beneficial effect on glucose metabolism independent of renin-angiotensin system (RAS) blockade. (Taylor et al., 2011). It activates PPAR- $\gamma$ at concentrations achieved in plasma following doses used for hypertension (Kurtz and Pravenec, 2004) and influences the expression of PPAR- $\gamma$ target genes involved in carbohydrate and lipid metabolism (Benson et al.,2004).

The aim of the present study was to investigate the effects of telmisartan and captopril on body mass index (BMI), homeostasis model assessment of insulin resistance (HOMA-IR), quantitative insulin sensitivity check index (QUICKI), fasting serum glucose (FSG) and fasting serum insulin (FSI) in T2DM hypertensive patients.

\section{MATERIALS AND METHOD}

This study, an open label design study, was conducted in the Department of Pharmacology, Mosul College of Medicine, University of Mosul and Al-Wafa Center in Mosul from 1 March, 2012 to 30 March, 2013. During that period, Wafa center attendants were screened for potential cases to enroll in the study, among those eligible who were commenced on therapy, only 68 diabetic hypertensive patients without any exclusion criteria continued with the study's antihypertensive regimen. Patients were randomly assigned to receive either telmisartan or captopril. Thirty four patients (18 male, 16 female) whose ages were between (36 and 80 years), received telmisartan (Telmi ${ }^{\circledR}$, Diamond Pharma, Syria), in a dose of $80 \mathrm{mg}$ once daily for two months. Thirty four patients (19 male, 15 female) whose ages were between 38 and 70 years, received captopril (Rilcapton $®$, Medochemie, Cyprus) in a dose of $50 \mathrm{mg}$ twice daily for 2 months. Forty T2DM normotensive patient, (21 males and 19 females), whose ages were between 35 and 74 years, matched for sex, age and BMI with the diabetic hypertensive patients groups, were taken as a control group.

To be eligible, T2DM patients should be type 2 diabetics maintained on a stable dose of hypoglycemic agents prior to and throughout study period, have HT either as newly diagnosed or the patient already diagnosed with HT, but not taking any antihypertensive drugs for at least 2 month prior to commencing antihypertensive treatment. The oral hypoglycemic agents were continued unchanged during the two- months study period. Patients with type 1 diabetes mellitus, those treated with thiazolidinediones, insulin or statins were excluded from the study.

BMI, FSG and FSI were measured prior to commencing antihypertensive therapy (Time zero) and after completing 2 months of treatment. The BMI was calculated as the weight in kilograms divided by the square of height in meters (Odegaard et al., 2009).

Obesity is defined as a BMI of $>30 \mathrm{~kg} / \mathrm{m}^{2}$ (Gordon et al., 2010). Five $\mathrm{ml}$ of venous blood was collected from each patient, using disposable syringe at about 8:30 to 10 A.M. after overnight fasting. The blood was allowed to clot in a gel separator tube (SGS Green VacTube, Standard Plus \& Medical Co., Ltd., Korea) at room temperature, and then the serum was separated by centrifugation at 3000 rpm for $10 \mathrm{~min}$, serum glucose was estimated by glucose-oxidase peroxidase colorimetric method (Lotta \& Turner, 1975) using a kit supplied by Randox Company (England).

Fasting serum insulin was measured by enzyme linked immune sorbent assay (ELISA) technique, using the Insulin AccuBind ${ }^{\circledR}$ ELISA KIT manufactured by Monobind, Inc. (USA). Insulin resistance was assessed according to HOMA-IR , and QUICKI.

HOMA-IR was calculated according to the following equation:

HOMA-IR $=$ fasting insulin $(\mu \mathrm{U} / \mathrm{ml}) \mathrm{x}$ FBG (mg/dl)/405 (Esteghamati, et al., 2010).

For an individual with "normal" insulin sensitivity, HOMA-IR=1(Muniyappa et al., 2008). Patients were considered as insulin 
resistant when HOMA $\geq 2.6$ (McAuley et al., 2001).

Quantitative insulin sensitivity check index was calculated as:

QUICKI $=1 /[\log$ FI $(\mu \mathrm{U} / \mathrm{ml})+\log$ FSG (mg/dl)] (Singh and Saxena, 2010).

Statistical analysis was performed using IBM SPSS Statistics for Windows, Version 22.0
(IBM Corp., Armonk, New York, USA). Paired and unpaired t-test as well as one-way ANOVA were used for analysis, with p-value $<0.05$ considered as the level of significance, data are presented as mean \pm standard deviation (SD).

\section{RESULTS}

Table (1). BMI and Age between the 3 groups (Mean \pm SD) (one-way ANOVA):

\begin{tabular}{|l|c|c|c|c|}
\hline & Telmisartan (n=34) & Captopril(n=34) & Control(n=40) & P-value \\
\hline BMI & $30.86 \pm 4.9$ & $31.02 \pm 5.42$ & $29.26 \pm 5.01$ & 0.259 \\
\hline Age & $52.85 \pm 9.75$ & $53.29 \pm 8.1$ & $51.15 \pm 8.99$ & 0.553 \\
\hline
\end{tabular}

Table (2). Baseline levels of studied parameters in all groups (Mean \pm SD) (one-way ANOVA):

\begin{tabular}{|l|c|c|c|c|}
\hline & Telmisartan $(\mathbf{n}=\mathbf{3 4})$ & Captopril(n=34) & Control(n=40) & P-value \\
\hline FSG $(\mathrm{mg} / \mathrm{dl})$ & $182.85 \pm 48.29$ & $187.32 \pm 65.43$ & $183.43 \pm 65.47$ & 0.946 \\
\hline QUICKI & $0.292 \pm 0.028$ & $0.293 \pm .024$ & $0.303 \pm 0.201$ & 0.077 \\
\hline FSI $(\mu \mathrm{U} / \mathrm{ml})$ & $18.58 \pm 10.01$ & $18.108 \pm 9.69$ & $12.74 \pm 4.92$ & $0.005^{*}$ \\
\hline HOMA-IR & $8.43 \pm 5.221$ & $8.27 \pm 6.283$ & $5.44 \pm 2.49$ & $0.012^{*}$ \\
\hline
\end{tabular}

* Significant difference for control versus both telmisartan and captopril groups at $<0.05$ level.

Table (3). Effect of Telmisartan therapy on study parameters (Mean \pm SD):

\begin{tabular}{|l|c|c|c|}
\hline & Before therapy & After 2 month & p-value \\
\hline FSG $(\mathrm{mg} / \mathrm{dl})$ & $182.85 \pm 48.3$ & $174.12 \pm 58.13$ & 0.358 \\
\hline QUICKI & $0.292 \pm 0.028$ & $0.299 \pm 0.034$ & 0.052 \\
\hline FSI $(\mu \mathrm{U} / \mathrm{ml})$ & $18.58 \pm 10.01$ & $17.06 \pm 9.38$ & 0.354 \\
\hline HOMA-IR & $8.43 \pm 5.22$ & $7.52 \pm 5.01$ & 0.324 \\
\hline BMI & $30.86 \pm 4.9$ & $30.65 \pm 5$ & 0.151 \\
\hline
\end{tabular}

Table (4). Effect of Captopril therapy on study parameters (Mean \pm SD):

\begin{tabular}{|l|c|c|c|}
\hline & Before therapy & After 2 month & p-value \\
\hline FSG $(\mathrm{mg} / \mathrm{dl})$ & $187.32 \pm 65.43$ & $162.95 \pm 56.87$ & $0.048^{*}$ \\
\hline FSI $(\mu \mathrm{U} / \mathrm{ml})$ & $18.107 \pm 9.69$ & $15.14 \pm 7.49$ & $0.022^{*}$ \\
\hline HOMA-IR & $8.27 \pm 6.28$ & $6.02 \pm 3.73$ & $0.027^{*}$ \\
\hline QUICKI & $0.293 \pm 0.024$ & $0.306 \pm 0.031$ & $0.022^{*}$ \\
\hline BMI & $31.03 \pm 5.42$ & $31.22 \pm 5.45$ & 0.18 \\
\hline
\end{tabular}

* Significant difference at $<0.05$ level

Table (5). Unpaired t-test for difference in (mean \pm SD) of variables before and after 2 months treatment between telmisartan and captopril group.

\begin{tabular}{|l|c|c|c|}
\hline & Telmisartan group & Captopril group & p-value \\
\hline FSG $(\mathrm{mg} / \mathrm{dl})$ & $-8.73 \pm 54.69$ & $-24.36 \pm 69.05$ & 0.304 \\
\hline QUICKI & $0.0066 \pm 0.019$ & $0.0133 \pm 0.029$ & 0.263 \\
\hline FSI $(\mu \mathrm{U} / \mathrm{ml})$ & $-1.51 \pm 9.4$ & $-2.96 \pm 7.17$ & 0.476 \\
\hline HOMA-IR & $-0.907 \pm 5.28$ & $-2.25 \pm 5.67$ & 0.315 \\
\hline BMI & $-0.2 \pm 7.93$ & $0.194 \pm 8.27$ & 0.841 \\
\hline
\end{tabular}




\section{DISCUSSION}

The patients in this study were both obese $\left(B M I \geq 30\left(\mathrm{~kg} / \mathrm{m}^{2}\right)\right.$ and insulin -resistant (HOMA $\geq 2.6$ ). It is known that in obese individuals, adipose tissue releases increased amounts of nonesterified fatty acids, glycerol, hormones, proinflammatory cytokines and other factors that are involved in the development of IR. (Steven et al., 2006).

There were non-significant differences regarding the age and BMI between the 3 studied groups at the initiation of the study (Table 1). There were significantly lower values for the control group versus baseline values of captopril and telmisartan groups in terms of FSI (Telmisartan group $=18.58 \pm 10.01$, Captopril group $=18.108 \pm 9.69$, control group $=12.74 \pm 4.92$, $\mathrm{p}=0.005$ ), and HOMA-IR (Telmisartan group $=8.43$ \pm 5.221 , Captopril group $=8.27 \pm 6.283$, control group $=5.44 \pm 2.49, \mathrm{p}=0.012$ ), while the 3 groups were non-significantly different in FSG and QUICKI index. (Table 2).

There were non-significant differences when comparing mean differences of study variables, achieved by telmisartan versus captopril, (Table 5) using unpaired t-test; mean difference was defined as (mean \pm SD of study parameter after 2 months therapy - mean $\pm \mathrm{SD}$ of baseline value).

The current study findings indicate that 2 months treatment with telmisartan produced nonsignificant differences with respect to FSG, FSI, HOMA-IR, QUICKI and BMI, versus baseline (initial) values in T2DM patients with HT (Table 3), while FSI, FSG and HOMA-IR decreased significantly (from $18.1 \pm 9.69$ to $15.14 \pm 7.49$, $\mathrm{p}=0.022$; and from $187.32 \pm 65.34$ to $162.95 \pm$ $56.87, \mathrm{p}=0.048$; and from $8.27 \pm 6.28$ to $6.02 \pm$ 3.73, $\mathrm{p}$-value $=0.027$, respectively) and QUICKI increased significantly (from $0.293 \pm 0.024$ to $0.306 \pm 0.031, p$-value $=0.022)$ in the captopril group versus baseline values (Table 4).

In agreement with these findings are those of Derosa et al. (2004a), who reported that after 12 months of therapy with telmisartan there were no significant changes from baseline in FSG, FSI, or HOMA-IR. Derosa et al. (2004b) studied 119 patients with mild essential hypertension and T2DM for 12 months; the administration of telmisartan (40 $\mathrm{mg}$ daily), eprosartan $(600 \mathrm{mg}$ daily), or placebo for 6 and 12 months did not result in any changes in FSG, FSI, or HOMAIR compared with baseline.

Derosa et al. (2007) evaluated 188 T2DM patients with metabolic syndrome receiving telmisartan $(40 \mathrm{mg} /$ day $)$ or irbesartan (150 $\mathrm{mg}$ /day), significant decreases in FSG were observed after 6 and 12 months in the telmisartan group, FSI showed a significant decrease at 12 months, and HOMA index showed significant improvement at 12 months in both groups, versus baseline. Such findings denote a possible long-term improvement in insulin sensitivity regarding telmisartan, it possible that telmisartan non-significant effect on IR in the present work thus is related to short duration of study. The patients in Derosa et al. (2007), however, were taking rosiglitazone, receiving special diet therapy and undergoing physical activity program during study period, all these may influence IR and could contribute to the observed effects on IR.

Kakuma et al. (2010) examined effects of telmisartan in 32 patients with T2DM, at $40 \mathrm{mg}$ per day for 6 months, increased to $80 \mathrm{mg}$ per day as required ; FSG was significantly lower at 6-month vs. 3-month and vs. baseline, FSI and HOMA-IR did not show statistically significant differences, again confirming neutral effects on IR in hypertensive diabetic population. In accordance with these findings, Nakayama et al. (2008) studied 20 patients with T2DM, after 8 weeks of olmesartan/telmisartan treatment (a crossover study design), no significant changes were reported in the patients.

Huang et al. (2011) reported no significant change compared with baseline in FSG, FSI (coinciding with current study findings), after therapy with telmisartan or losartan, except for HOMA-IR, which was improved by telmisartan, in obese hypertensive patients. Again in agreement with our findings, Ozaki et al. (2010) reported that FSI, HOMA-IR and FSG were non- significantly changed versus baseline in 74 hypertensive T2DM patients receiving telmisartan. Also in agreement with current study findings, Bahadir et al. (2007) found that both telmisartan or losartan treatment had a neutral effect on IR in hypertensive and metabolic syndrome patients. In accordance with these findings, Wohl et al. (2010) failed to show a statistically significant effect of telmisartan on insulin sensitivity, despite using the hyperinsulinemic clamp technique to assess insulin sensitivity, which is the gold standard method for IR assessment, and this was consistent with findings of Usui et al. (2007). All these results indicate the non-significant effect of telmisartan on FSG, FSI and insulin resistance, as demonstrated in current work.

In contrast to our findings, however, Miura et al. (2005) studied 18 hypertensive T2DM patients treated with valsartan $(80 \mathrm{mg} /$ day $)$ or candesartan $(8 \mathrm{mg} /$ day $)$ for 6 months. Then, medication changed to telmisartan (40 mg/day) for 
12 weeks. Telmisartan treatment resulted in a significant decrease in FSI level, decreases in FSG were not statistically significant. However, our patients had no previous ARB treatment history which could have caused a "carry over" effect to the period of telmisartan treatment in Miura's study (2005).

Vitale et al. (2005) reported that compared with losartan, telmisartan (80 $\mathrm{mg}$ daily) for hypertension for 3 months, significantly reduced FSG, FSI and HOMA-IR. However, the patients were non-diabetic with metabolic syndrome. Another study (Yamada et al., 2008) showed that telmisartan treatment for 3 months improved serum levels of glucose better than candesartan in hypertensive diabetic patients but with nonsignificant changes from baseline in FSG and FSI.

Watanabe et al. (2010) suggested that while many clinical studies have focused on the metabolic effects of telmisartan, results obtained are not consistent. He suggested that this discrepancy may be explained by differences in study population background factors including race, BMI and glycemic control levels, noting that earlier studies are limited by relatively small patient numbers and treatment durations. One possible explanation of the discrepancy in previous studies findings, suggested by Usui et al. (2007), is that telmisartan improves insulin resistance only in early stage diabetic patients, who do not need insulin injections or sulfonylurea for their glycemic control, thus telmisartan could either be neutral or have a positive impact on insulin sensitivity, depending on the population studied, accordingly, telmisartan may have failed in the present work to improve IR, possibly due to substantial metabolic derangement in the study group.

In agreement with the current study findings, ACE inhibition has been shown to improve insulin sensitivity and glycaemic control in diabetic patients, it also resulted in a $14 \%$ relative reduction in the incidence of new-onset T2DM in the Captopril Prevention Project (CAPPP) (Prasad and Quyyumi, 2004).

The ACE inhibitors have an established beneficial effect on insulin sensitivity. The ACEIs have been shown to reduce IR associated with arterial HT (Otero, 2007). It has been suggested that the RAS, kallikrein-kinin system, or both participate in this effect, whose precise mechanism, however, has yet to be elucidated (Derosa et al. ,2003).

In contrast, Tillmann et al. (1997) reported that long-term (12 month) low-dose ACEIs (captopril and enalapril) did not modify insulin sensitivity and glycaemic control in normotensive T2DM subjects. Similar non-significant changes in
T2DM hypertensive patients were reported by Romero et al. (1990), and again by Romero et al. (1992), but very small sample size (10 patients) is a noteworthy limitation of these 2 studies. Similarly, Rett et al. (1988) reported unaltered FSI after a 3-week study of captopril in 30 T2DM patients, probably the short period of study is a drawback to validate their findings. Similarly a 3 month treatment of captopril in 14 hypertensive diabetics resulted in fasting plasma glucose, insulin, insulin sensitivity and glycosylated hemoglobin unchanged from baseline (Yin et al., 1994). Also, Giordano et al. (1995) reported no improvement in insulin action after 3-month captopril treatment, consistent with other previous studies in hypertensive diabetic subjects.

In contrast, Jasik et al. (1993) reported that after 2-week captopril therapy in both non-diabetic and diabetic hypertensives, lower values of glycated hemoglobin and FSI were found. Similarly, Torlone et al. (1993) reported that captopril resulted in greater insulin sensitivity in hyperglycemic conditions, greater insulin sensitivity in euglycemic conditions at liver, muscle, adipose tissue level after 3 month period in diabetic hypertensive patients. Also Alkharouf et al. (1993) reported that comparing before and at 1 , 2,3 , and 4 months of captopril monotherapy, there were significant reductions in FSG from baseline at 1 month $(P<.01)$ and further stepwise decreases in values at 2,3 , and 4 months, indicating improved insulin sensitivity in T2DM hypertensive patients.

Despite the fact that Telmisartan did not, unlike captopril, decrease IR in the current study, it should be stressed that RAS blockade is reported to reduce IR, which is the pathophysiological hallmark of the metabolic syndrome and T2DM. Better skeletal muscle perfusion, improvement of microvascular changes, and increased perfusion of the pancreatic islet cell are some of the proposed mechanisms by which insulin sensitivity is increased (Patel and Mehta, 2012).

Additionally, direct effects of angiotensin II on the pancreatic $\beta$ cells from a local RAS in the islet might contribute to a loss of $\beta$ cell function. Activation of RAS is associated with fibrosis of pancreatic islets in animals with T2DM (Patel and Mehta, 2012).

Although there remains some controversy as to whether the ACE inhibitors enhance insulin sensitivity in diabetic subjects, previous studies did not demonstrate a deleterious effect of this class of drugs on glucose tolerance and insulin sensitivity. (Giordano et al., 1995).

Another measure of IR, QUICKI, was studied in the present work. Since it is an inverse 
of the logarithm of product of (FSI * FSG), it runs in the opposite direction of HOMA-IR (i.e. increased value implies less IR). It has been shown to provide a consistent and precise index of insulin sensitivity with better positive predictive power, and has also been shown to have a better linear correlation with glucose clamp determination (the gold standard method) than other methods, especially in obese and diabetic subjects (Singh and Saxena, 2010).

It is possible that the beneficial metabolic effects of ACEIs go well beyond just simple interruption of the RAS, they might improve glucose metabolism through effects on kinin-nitric oxide pathways as demonstrated by studies with bradykinin receptor knockout mice and with bradykinin antagonists (Kurtz and Pravenec, 2004).

\section{CONCLUSIONS}

Captopril produces significant improvement in IR, in T2DM hypertensive patients, while corresponding effect of telmisartan is insignificant.

\section{RECOMMENDATIONS}

Since metabolic adverse effects were not demonstrated by either treatments, in hypertensive T2DM patients, both drugs can be recommended for such patients. Since captopril produced a more favorable influence on IR, it may be recommended over telmisartan for intial antihypertensive therapy, however, more frequent dosing of captopril (twice versus once daily for telmisartan) may discourage such patients producing patient non-compliance , telmisartan may thus be a better alternative if compliance cannot be guaranteed.

\section{REFERENCES}

Alkharouf, J.; Nalinikumari, K.; Cony, D. and Tuck, M.(1993). Long-term effects of angiotensin converting enzyme inhibitor captopril on metabolic control in noninsulin-dependent diabetes mellitus. $\mathrm{Am} J$ Hypertens. 6. P.p. 337-343.

Bahadir, O.; Uzunlulu, M.; Oguz, A. and Bahadir, M.A. (2007). Effects of telmisartan and losartan on insulin resistance in hypertensive patients with metabolic syndrome. Hypertens Res. 30. P.p. 49-53.

Benson, S.C.; Pershadsingh, H.A.; Ho, C.I.; Chittiboyina, A.; Desai, P.; Pravenec M.; Qi, N.; Wang, J.; Avery, M.A. and Kurtz, T.W. (2004). Identification of telmisartan as a unique angiotensin II receptor antagonist with selective PPAR $\gamma$-modulating activity. Hypertension. 43. P.p. 993-1002.
Berra, K. and Miller, N.H. (2009). Inhibiting the renin-angiotensin system: Why and in which patients. J Am Acad Nurse Pract. 21. P.p. 66-75.

Cohen, D.L. and Townsend, R.R. (2008, Oct. 10). What should the physician do when creatinine increases after starting an angiotensin-converting enzyme inhibitor or an angiotensin receptor blocker? $\mathrm{J} \mathrm{Cl}$ Hypertens. 10(10). P.p. 803-804.

Derosa, G.; Cicero, A.F.G.; Ciccarelli, L. and Fogari, R. (2003). A randomized, doubleblind, controlled parallel-group comparison of perindopril and candesartan in hypertensive patients with type 2 diabetes mellitus. Clin Ther. 25. P.p. 2006-2021.

Derosa, G.; Cicero, A.F.G.; Bertone, G.; Piccinni, M.N.; Fogari, E.; Ciccarelli, L.; and Fogari, R. (2004a). Comparison of the effects of telmisartan and nifedipine gastrointestinal therapeutic system on blood pressure control, glucose metabolism, and the lipid profile in patients with type 2 diabetes mellitus and mild hypertension: a 12-month, randomized, double-blind study. Clin Ther. 26 (8). P.p. 1228-1236.

Derosa, G.; Ragonesi, P.D.; Mugellini, A.; Ciccarelli, L.; and Fogari, R. (2004b). Effects of telmisartan compared with eprosartan on blood pressure control, glucose metabolism and lipid profile in hypertensive, type 2 diabetic patients: a randomized, double-blind, placebocontrolled 12-month study. Hypertens Res. 27 (7). P.p. 457-464.

Derosa, G.; Fogari, E.; D’Angelo, A.; Cicero, A.F.G.; Salvadeo, S.A.T.; Ragonesi, P.D.; Ferrari, I.; Gravina, A.; Fassi, R. and Fogari, R. (2007). Metabolic effects of telmisartan and irbesartan in type 2 diabetic patients with metabolic syndrome treated with rosiglitazone. J Clin Pharm Ther. 32. P.p. 261-268.

Esteghamati, A.; Ashraf, H.; Khalilzadeh, O.; Zandieh, A.; Nakhjavani, M.; Rashidi, A.; Haghazali, M. and Asgari, F. (2010). Optimal cut-off of homeostasis model assessment of insulin resistance (HOMA-IR) for the diagnosis of metabolic syndrome: third national surveillance of risk factors of non-communicable diseases in Iran (SuRFNCD-2007). Nutr Metab. 7. P.p. 26. 1-8.

Giordano, M.; Matsuda, M.; Sanders, L.; Canessa, M.L.; and DeFronzo, R.A. (1995). Effects of angiotensin-converting enzyme inhibitors, $\mathrm{Ca} 2+$ channel antagonists, and $\alpha$-adrenergic 
blockers on glucose and lipid metabolism in NIDDM patients with hypertension. Diabetes. 44. P.p. 665-671.

Gordon, L.; Ragoobirsingh, D.; Morrison, E.; McGrowder, D.; Choo-Kang, E. and Martorell, E. (2010, Oct). Dyslipidaemia in hypertensive obese type 2 diabetic patients in Jamaica. Arch Med Sci. 6(5). p.p. 701708.

Huang, G.Z.; Tang, Y.H.; Wang, B.Y.; Zhang, B.; Hu, T.J.; Zhang, L.; Zhang, X.Y. and Zhong, Y. (2011). Effects of telmisartan on insulin resistance and visceral fat distribution in Chinese hypertensive patients with obesity. Saudi Med J. 32 (10). P.p. 1017-1021.

Ibrahim, I.A.; Al-Joudi, F.S.; Suleiman, R.W. and Al-Saffar, B.H. (2010, Apr). Captopril interferes with some serum biochemical findings. Afr J Biochem Res. 4(4). P.p. 9598.

Kurtz, T.W. and Pravenec, M. (2004). Antidiabetic mechanisms of angiotensin-converting enzyme inhibitors and angiotensin II receptor antagonists: beyond the reninangiotensin system. J Hypertens. 22 (12). P.p. 2253-2261.

Lotta, J.A. and Turner, K. (1975). Evaluation of trinder's glucose oxidase method for measuring glucose in serum and urine. Clin Chem. 2. P.p. 1754-1760.

Mansour, A.A. (2012). Prevalence and control of hypertension in Iraqi diabetic patients: A prospective cohort study . Open Cardiovasc Med J. 6. P.p. 68-71.

Matheus, A.S.M.; Tannus, L.R.M.; Cobas, R.A.; Palma, C.C.S.; Negrato, C.A. and Gomes, M.B. (2013). Impact of Diabetes on Cardiovascular Disease: An Update. Int $J$ Hypertens. 2013. P.p. 1-15.

McAuley, K.A.; Williams, S.M.; Mann, J.I.; Walker, R.J.; Lewis -Barned, N.J.; Temple, L.A. and Duncan, A.W. (2001). Diagnosis insulin resistance in the general population. Diabetes Care. 24. p.p. 460-464.

McFarlane, S.I.; Banerji, M. and Sowers, J.R. (2001). Insulin resistance and cardiovascular disease. J Clin Endocrinol Metab. 86(2). p.p. 713-718.

Miura, Y.; Yamamoto, N.; Tsunekawa, S.; Taguchi, S.; Eguchi, Y.; Ozaki, N. and Oiso, Y. (2005). Replacement of valsartan and candesartan by telmisartan in hypertensive patients with type 2 diabetes: Metabolic and antiatherogenic consequences. Diabetes Care. 28 (3). P.p. 757-758.

Muniyappa, R.; Lee, S.; Chen, H. and Quon, M.J. (2008). Current approaches for assessing insulin sensitivity and resistance in vivo: advantages, limitations, and appropriate usage. Am J Physiol Endocrinol Metab. 294. P.p. E15-E26.

Odegaard, A.; Koh, W.P.; Vazquez, G.; Arakawa, K.; Lee, H.P.; Yu, M. and Pereira, M. (2009, Jun). BMI and Diabetes Risk in Singaporean Chinese. Diabetes Care. 32(6). p.p. 11041106.

Olivares-Reyes, A.; Arellano-Plancarte, A. and Castillo-Hernandez, J.R. (2009). Angiotensin II and the development of insulin resistance: Implications for diabetes. Mol Cell Endocrinol. 302. P.p. 128-139.

Östergren, J. (2007, May). Renin-angiotensinsystem blockade in the prevention of diabetes. Diab Res Clin Prac. 76(3). P.p. S13-S21.

Otero, M.L. (2007). Manidipine-delapril combination in the management of hypertension. Vasc Health Risk Manag. 3(3). P.p. 255-263.

Patel, B.M. and Mehta, A.A. (2012). Aldosterone and angiotensin: Role in diabetes and cardiovascular diseases. Eur $J$ Pharmacol. 697. P.p. 1-12.

Patel, B.M and Mehta, A.A. (2013, Sep). Choice of anti-hypertensive agents in diabetic subjects. Diab Vasc Dis Res. 10(5). P.p. 385-396.

Prasad, A. and Quyyumi, A.A. (2004). ReninAngiotensin System and Angiotensin Receptor Blockers in the Metabolic Syndrome. Circulation. 110. P.p. 15071512.

Rett, K.; Wicklmayr, M.; Tschollar, W.; Dietze, G. and Mehnert, H. (1988). Role of angiotensin-converting enzyme inhibitors in early antihypertensive treatment in noninsulin dependent diabetes mellitus. Postgrad Med J. 64 .Suppl 3. P.p. 6974.[Abstract].

Romero, R.; Salinas, I.; Teixidó, J.; Lucas, A.; Felip, A. and Sanmarti, A. (1990, Dec). Long term follow-up of the effect of captopril on severe proteinuria in hypertensive diabetic patients. $J$ Hum Hypertens. 4(6). P.p. 671-675.

Romero, R.; Salinas, I.; Lucas, A.; Teixidó, J.; Audi, L. and Sanmarti, A. (1992, Sep). Comparative effects of captopril versus nifedipine on proteinuria and renal function of type 2 diabetic patients. Diab Res Clin Pract. 17(3). P.p. 191-198.

Saini, V. (2010, Jul.).Molecular mechanisms of insulin resistance in type 2 diabetes mellitus. World J Diabetes. 1(3). P.p. 68-75. 
Savage, D.B.; Petersen, K.F. and Schulman, G.I. (2005). Mechanisms of insulin resistance in humans and possible Links with inflammation. Hypertension. 45. p.p. 828833.

Savage, D.B.; Petersen, K.F. and Schulman, G.I (2007). Disordered Lipid Metabolism and the Pathogenesis of Insulin Resistance. Physiol Rev. 87. P.p. 507-520.

Schutta, M.H. (2007). Diabetes and hypertension: epidemiology of the relationship and pathophysiology of factors associated with these comorbid conditions. $J$ Cardiometab Syndr. 2(2). P.p. 124-30.

Singh, B. and Saxena, A. (2010).Surrogate markers of insulin resistance: A review. World $J$ Diabetes. 1(2). P.p. 36-47.

Standl, E.; Erbach, M. and Schnell, O. (2012). What should be the antihypertensive drug of choice in diabetic patients and should we avoid drugs that increase glucose levels? Pro and Cons. Diabetes Metab Res Rev. 28 (Suppl. 2). P.p. 60-66.

Steven, E.; Rebecca, L. and Kristina, M. (2006). Review Article Mechanisms linking obesity to insulin resistance and type 2 diabetes. Nature. 444. P.p. 840-846.

Tillmann, H.C.; Walker, R.J.; Lewis-Barned, N.J.; Edwards, E.A. and Robertson, M.C. (1997, Aug). A long-term comparison between enalapril and captopril on insulin sensitivity in normotensive non-insulin dependent diabetic volunteers. $J$ Clin Pharm Ther. 22(4). p.p. 273-278.

Torlone, E.; Britta, M.; Rambotti, A.M.; Perriello, G.; Santeusanio, F.; Brunetti, P. and Bolli, G.B. (1993). Improved insulin action and glycemic control after long-term angiotensin-converting enzyme inhibition in subjects with arterial hypertension and type
II diabetes. Diabetes Care. 16. P.p. 13471355.

Taylor, A.A.; Siragy, H. and Nesbitt, S. (2011). Angiotensin receptor blockers: Pharmacology, efficacy, and safety. J Clin Hypertens (Greenwich). 13(9). P.p. 677686.

Vitale, C.; Mercuro, G.; Castiglioni, C.; Cornoldi, A.; Tulli, A.; Fini, M.; Volterrani, M. and Rosano, G.M.C. (2005). Metabolic effect of telmisartan and losartan in hypertensive patients with metabolic syndrome. Cardiovasc Diabetol. 4. P.p. 6-10.

Wohl, P.; Krusinova, E.; Hill, M.; Kratochvilova, S.; Zıdkova, K.; Kopecky, J.; Neskudla, T.; Pravenec, M.; Klementova, M.; Vrbıkova, J.; Wohl, P.; Mlejnek, P. and Pelikanova, T. (2010). Effect of telmisartan on selected adipokines, insulin sensitivity, and substrate utilization during insulin-stimulated conditions in patients with metabolic syndrome and impaired fasting glucose. Eur $J$ Endocrinol. 163(4). P.p. 573-583.

Yamada, S.; Ano, N.; Toda, K.; Kitaoka, A.; Shiono, K.; Inoue, G.; Atsuda, K.; and Irie, J. (2008). Telmisartan but not candesartan affects adiponectin expression in vivo and in vitro. Hypertens Res. 31. P.p. 601-606.

Yin, W.; Seghieri, G.; Boni, C.; Sanna, G.; Anichini, R.; Bartolomei, G. and Ferrannini, E. (1994, Mar). Effect of chronic ACE inhibition on glucose tolerance and insulin sensitivity in hypertensive type 2 diabetic patients. Chin Med Sci J. 9 (1). P.p. 29-33. 